\title{
EL PERSONAL DIRECTIVO PÚBLICO A LA LUZ DE LA ÚLTIMA REFORMA DE LA ADMINISTRACIÓN PÚBLICA ITALIANA Y TRAS LA SENTENCIA N. 251/2016 DEL TRIBUNAL CONSTITUCIONAL
}

\author{
Giuseppina Pensabene Lionti \\ Dottore di ricerca in Diritto Privato (Sez. Dinitto del Lavoro) \\ Università degli Studi di Palemo \\ Doctora en Derecho (Dpto. Derecho del Trabajo y de la Seguridad Social) \\ Universidad de Valencia \\ Professo re a contratto (IUS/07),Università degli Studi di Palemo \\ Avvocato
}

\section{Resumen}

Las normas contenidas en la Ley n. 124/2015 ("Reforma Madia") abren un nuevo período de reformas del empleo público italiano, según los (proclamados) principios de simplificación, flexibilidad y unidad. Las previsiones concernientes al personal directivo público contenidas en el art. 11 de la citada Ley y en el esquema de decreto legislativo aprobado el 26 agosto de 2016 suscitan especial interés. Entre ellas, destacan las relativas a la unificación de los "ruoli dirigenziali" y a la abolición de las "due fasce"; no sólo por su alcance innovador, sino también por las conexas consecuencias que de ellas derivan y que se reflejan tanto en las modalidades de selección de dicho colectivo (aún en el ámbito de la especificidad de cada una de las "ramas" de la Administración Pública), como en la asignación del puesto y en el sistema de atribución de los cargos directivos. Se señalan pues los aspectos de mayor criticidad de la nueva

- Palabras clave: Personal; Directivo; Público; Reforma; Ordenamiento; Jurídico; Italiano; Empleo; Cargo; Comisión; Independiente; Responsabilidad; Gerencial; Acceso; Unificación; Sector; Sistema de botín; Cese; Discrecionalidad; Política; Administración; Acceso; Función; Oposición; Concurso; Formación; Tribunal Constitucional regulación, sobre todo bajo el perfil de las responsabilidades, de la eficacia de las funciones públicas y de la armonía constitucional; pudiendo derivar este "hipernormativismo" en una disminución de tutelas efectivas. Todo ello sin abandonar un enfoque critico sobre la "evanescencia" de dicha reforma que todavía no ha entrado en vigor, puesto que el Tribunal Constitucional italiano, con la sentencia n. 251 del 25 de noviembre de 2016, ha declarado la inconstitucionalidad, entre otras normas, del citado art. 11. 
- Keywords:

Public; Directive;

Management; Reform;

Jobs Act; Heads;

Administration;

Flexibility;

Simplification;

Duration;

Constitutional; Court;

Italian; Law; System;

Labour; Employment
Abstract

This paper examines the provisions contained in Law $\mathrm{n}$. $124 / 2015$, that opened a new season of reforms for the Italian public labour, in accordance with the (declaimed) principles of simplification, flexibility and unity. It is worth highlighting the legal framework provided by article 11 and by the decree law approved last August, in particular focusing on the unification of directive roles and the abolition of the two executive classes. The innovative character of the recently mentioned article (which was affected by the n. 251/2016 ruling of the Constitutional Court), has also affected the process for the selection of executives in the different areas of the Public Administration; hence, the new norms have adjusted the procedure for the assignment of roles and entrustment of the heads of the Administration. All of this it is examined without leaving a critical view of this reform that has not been approved yet.

I. La posición central ocupada por el personal directivo en el atormentado proceso de reforma del empleo público italiano - II. La unificación del sector directivo - III. Nuevas reglas para la atribución del cargo directivo y el papel crucial de la Comisión independiente - IV. Apuntes sobre el nuevo sistema de acceso a la función directiva - V. Algunos "retoques" al sistema de responsabilidad gerencial de los directivos públicos - VI. A modo de conclusión - Bibliografía

\section{LA POSICIÓN CENTRAL OCUPADA POR EL PERSONAL DIRECTIVO EN EL ATORMENTADO PROCESO DE REFORMA DEL EMPLEO PÚBLICO ITALIANO}

El empleo público italiano ha sido objeto de reformas continuas, que siempre han interesado, directamente o indirectamente, el personal directivo. De hecho, en todos los intentos de innovación y modernización administrativa que se han puesto en marcha, la dirigencia pública ha ocupado una posición central, repensándose su régimen jurídico plúrimas veces. Tras la "privatización del empleo público"1 de los

${ }^{1}$ A grandes rasgos, la privatización del empleo público italiano planteó la reestructuración de la dirección pública en clave empresarial, extendiendo las normas de derecho laboral privado (tanto legales como estatutarias) y las fuentes contractuales (tanto individuales como colectivas) al sector público del trabajo. En consecuencia, el contencioso judicial en materia fue sustraído de la competencia del juez administrativo y dirigido a la del juez de lo social. Sobre dicho fenómeno la bibliografía es muy extensa; se recuerdan, ex plurimis, los estudios de: GARILLI, A., "La privatizzazione del lavoro nelle pubbliche amministrazioni e l'art. 97 Cost.: di alcuni problemi e dei possibili rimedi", en Riv. Giur. Lav., I, 2007, 301 ss.; BATTINI, S., CASSESE, S. (a cura de), Dall'impiego pubblico al rapporto di lavoro con le pubbliche amministrazioni, Milano, 1997; LISO, F., "La privatizzazione dei rapporti di lavoro", en CARINCI, F. (dirigido por), Il lavoro alle dipendenze delle amministrazioni pubbliche (dal d.lgs. 29/1993 alla finanziaria del 1995). Commentario, Milano, 1995, p.73 e ss; CLARICH, M., IARIA, D., La riforma del pubblico impiego, Rimini, 1994; D’ORTA, C., 
años noventa y las reformas "Frattini" del $2002^{2}$ y "Brunetta" del $2009^{3}$, la llamada "reforma Madia" de reorganización de las Administraciones Públicas (Ley n. 124/2015) -todavía en desarollo- puede ser considerada la "cuarta fase" del atormentado proceso reformador del sector público del derecho del trabajo italiano ${ }^{4}$.

Así pues, en Italia las reformas se suceden una detrás de la otra con ese carácter parcial y fragmentario, volviendo siempre a plantearse la necesitad de delinear un espacio directivo dentro de la función pública dotado de una serie de rasgos profesionales que lo singularizan tanto frente a la actividad estrictamente política, como frente a las tareas meramente burocráticas desempeñadas por los funcionarios. En efecto, los altos directivos públicos sí forman parte de la burocracia administrativa, pero son "burócratas profesionales" a los que se requiere una implementación eficaz y eficiente para servir mejor a los intereses públicos 5 .

A la luz de lo dicho, la disciplina italiana de los directivos públicos constituye una temática trascendente. Ello es corroborado por el hecho de que, hasta la actualidad, la legislación del Estado no ha resuelto de modo satisfactorio cuestiones siempre pendientes como, por ejemplo, el problema de la ordenación de las relaciones entre Politíca y Administración que se halla detrás del régimen jurídico del personal directivo público y de su conexión con el debate acerca de la auspiciada "modernización administrativa".

La última reforma de la Administración Pública, aprobada bajo el Gobierno Renzi, pretende afrontar estas dificultades y buscar las pertinentes soluciones para solventar los diversos aspectos problemáticos que tradicionalmente han caracterizado a las

MEOLI, C., La riforma della dirigenza pubblica, Padova, 1994; CECORA, G., D’ORTA, C., La riforma del pubblico impiego, Bologna, 1993; ZOPPOLI, L., "La dirigenza nel pubblico impiego "privatizzato" ”, en RUSCIANO, M. -ZOPPOLI, L. (a cura de), L’impiego pubblico nel diritto del lavoro, Torino, 1993; CASSESE, S., "Il sofisma della privatizzazione del pubblico impiego", en Riv. it. dir. lav., I, 1993; DI CERBO, V., "La cosiddetta "privatizzazione del pubblico impiego" ", en Lav. prev. oggi, 1993; VIRGA, P., Il pubblico impiego dopo la privatizzazione, Milano, 1993; GHEZZI, G., "La legge delega per la riforma del pubblico impiego", en Riv. Giur. Lav., I, 1992; GIANNINI, M. S., "Per la privatizzazione del rapporto di pubblico impiego", en Scritti in onore di Mario Nigro, II, Milano, 1991, p. 168 e ss.

2 Sobre la reforma introducida por la Ley n. 145/2002 ("reforma Frattini"), vid., por ejemplo: GARILLI, A., "Profili dell'organizzazione e tutela della professionalità nelle pubbliche amministrazioni", en Giorn. Dir. Lav. Rel. Ind., 2004; VALENSISE, B., La dirigenza statale. Alla luce delle disposizioni normative contenute nella legge 15 luglio 2002, n. 145, Torino, 2002; D'ORTA, C., "Gli incarichi dirigenziali nello Stato dopo la legge 145/2002", en LPA, 2002, p.935 e ss.

${ }^{3}$ Por todos, vid. NAPOLI, M. - GARILLI, A., La terza riforma del lavoro pubblico tra aziendalismo e autoritarismo, Padova, 2013.

${ }^{4}$ BOSCATI, A., "La politica del governo Renzi per il settore pubblico tra conservazione e innovazione: il cielo illuminato diverrà luce perpetua?", en LPA, 2014, n.2, pp. 233 e ss.

5 En esta linea, es specialmente significativo el diseño institucional delineado por los artículos 97 y 98 de la Constitución Italiana; vid., por todos, PINELLI, C., "Commento agli artt. 97 e 98”, en BRANCA, G.- PIZZORUSSO, A. (dirigido por), Commentario alla Costituzione, Bologna-Roma, 1994. 
Administraciones públicas italianas, in primis: exceso de formalización, falta de distinción entre tareas a desempeñar y poca adecuación entre las pruebas selectivas de acceso a la función publica.

Sin embargo, entregarse a estas presiones es una forma evidente de seguir en la pretendida búsqueda de un merit system (que todavía no puede considerarse alcanzado), a través del cual se pueda implantar finalmente un modelo de gestión pública plenamente profesional y armonizar las dos "almas contrapuestas" que conviven dentro de ello: management y governance ${ }^{6}$.

Así bien, la citada Ley n. 124/2015, en su artículo 11, dispone una revisión radical de la disciplina jurídica del personal directivo público ${ }^{7}$, la cual, aunque esté formalmente inspirada en principios de simplificación, flexibilidad y unidad, parece caminar más bien hacia una mayor precarización de dicho colectivo.

De otra parte, y según lo señalado anteriormente, para poder delinear un cuadro completo del nuevo diseño reformador será necesario esperar la emanación de los decretos de actuación de la Ley n. 124/2015, la cual, por el momento, sólo reúne una serie de principios y criterios directivos, reenviando el concreto desarrollo de los mismos a los órganos de Gobierno.

En efecto, aunque en el dia 26 de agosto de 2016, el Consejo de los Ministros ha aprobado un esquema de decreto legislativo sobre la disciplina de la "Dirigenza della Repubblica" 8 -para completar el cuadro- sobre dicho decreto han sido elaborados los dictamenes del Consiglio di Stato (el 14 de octubre de 2016), de la Conferenza Unificata (el 3 de noviembre de 2016) y de las Commissioni Affari Costituzionali de la Camera y del Senato (respectivamente, el 9 y el 10 de noviembre); la Corte Costituzionale, con la sentencia n. 251 de 25 de noviembre de 2016, ha declarado la incostitucionalidad, entre otras normas, del citado art. $11^{9}$.

Así pues, la nueva normativa todavía no ha entrado en vigor.

Además, como es bien sabido, el resultado del referendum constitucional que hubo en Italia el 4 de diciembre de 2016 llevó a cabo el cambio de Gobierno, pues el ministro Gentiloni pasa a ser el Presidente del Consejo de los Ministros italiano, en lugar de

${ }^{6}$ Para un análisis, también en una perspectiva de derecho comparado, de los proyectos de reforma de las Administraciones Públicas inspirados al concepto del new public management, vid. POLLIT, C.- BOUCKAERT, G., Public Management Reform, Oxford, 2011; y, también, sobre el New Public Management, basado en los principios "empresariales", vid. KICKERT, W.M., (dirigido por), The Study of Public Management in Europeand the US, London, 2008.

${ }^{7}$ DEODATO, C., "Brevi riflessioni sulla costituzionalità e sulla ragionevolezza di alcuni aspetti della riforma della dirigenza pubblica", en $L P A, 2014$, n. 6, p. 946; en especial, el Autor subraya que es difícil destacar un verdadero continuum entre la disciplina vigente y la anterior.

${ }^{8}$ Dicho decreto es consultable en www.funzionepubblica.gov.it.

${ }^{9}$ Sobre todo ello, sea consentido un reenvío a PENSABENE LIONTI, G, "La riforma della dirigenza pubblica: prospettive ambiziose e risultati deludenti”, en www.adapt.it, 9 de enero de 2017. 
Renzi. Todo ello aumenta la incertidumbre in subiecta materia, puesto que no es seguro que el nuevo Gobierno quiera seguir con la reforma de la Administración Pública, pudiendo derivar esta ambigüedad en una verdadera caída al vacio de la debatida "reforma Madia" sobre el personal directivo público ${ }^{10}$.

No obstante, el decisum de la citada sentencia constitucional no censura la "sustancia" de las normas dictadas en materia de personal directivo, sino que se limita a evidenciar una violación procedimental concerniente a la fase de mera elaboración del esquema de decreto legislativo del 26 de agosto de 2016, que -vulnerando el principio constitucional general de "leal colaboración"- no ha tenido en cuenta la oportunidad de realizar, antes de la emanación de los decretos, un acuerdo previo con las Regiones, a las que la normativa iba a extender su propio ambito de aplicación.

Estando así las cosas, "el efecto tsunami” producido por la sentencia de qua, que no ha permitido que dicha normativa entrase en vigor, podría ser absordido, ex post, por una nueva Ley y por nuevos decretos que, manteniendo lo regulado a nivel sustancial por la reforma, corrijan los errores cometidos bajo el perfil procedimental ${ }^{11}$; lo que parece -por lo menos formalmente- corroborado por el reciente dictamen del Consiglio di Stato del 9 de enero de 2017.

Así bien, es muy probable que, a pesar de todo lo dicho, la nueva disciplina sobre el personal directivo público, pueda sobrevivir bajo el nuevo Gobierno Gentiloni. Y, sin duda alguna, es posible prefigurar l'an del nuevo régimen jurídico de los directivos públicos y destacar las líneas maestras sobre las que se asienta el modelo actual de personal directivo público italiano, al margen de los modelos concretos.

A la luz de todo lo expuesto, este trabajo, sin ninguna pretensión de exhaustividad, se propone apuntar solo algunas de las novedades mayormente impactantes introducidas por la última reforma de la Administración Pública italiana: la unificación del sector directivo; el nuevo régimen de atribución de cargos directivos; la institución de una "Comisión independiente para la dirigencia"; el sistema de acceso a la función directiva, y las nuevas reglas previstas en materia de responsabilidad gerencial.

\section{LA “UNIFICACIÓN” DEL SECTOR DIRECTIVO}

Una de las novedades más significativas previstas por la "reforma Madia" respecto a la normativa anterior contenida en el D.lgs. 165/2001 (Testo Unico del Pubblico Impiego), consiste en la unificación del sector directivo (ruolo unico) de todas las Administraciones Públicas (tanto la estatal como las territoriales) de manera que, una vez superada la oposición -según los principios generales establecidos por el último apartado del art. 97 Cost. y las reglas específicas previstas por los arts. 28 y ss. del citado D.lgs. 165/2001- se puedan atribuir cargos directivos a cualquier dirigente

10 MARSHALL, J., "La Corte Costituzionale, senza accorgersene, modifica la forma di Stato?", en Giorn. dir. amm., 6/2016, p. 710.

11 En este sentido, vid. FERRANTE, V., "Fare i conti senza l'oste: le Regioni, la riforma Madia e la Corte Costituzionale", en www.ipsoa.it, 3 de diciembre de 2016. 
público, que presente los requisitos de mérito y capacidad necesarios para el desempeño de dichas funciones ${ }^{12}$.

La ratio de tal previsión reside basicamente en la voluntad de asegurar una plena movilidad entre las distintas áreas de la Administración Pública a la que se acompaña la configuración de un sistema de formación continua ${ }^{13}$, considerada como una herramienta central para el desarrollo de las capacidades directivas ${ }^{14}$. Además, se propone introducir una especie de "base de datos de las competencias"15, que pueda recoger todas las capacidades, potencialidades, competencias, especialidades de los candidatos con la finalidad de seleccionar, entre ellos, a quien presente el perfil exacto del puesto directivo que se pretende cubrir.

Sin duda dichas previsiones pueden ser abordadas desde múltiples y muy variados puntos de vista; lo cual, desde luego, tampoco contribuye a un mejor entendimiento de las mismas.

Según un primer orden de consideraciones, la unificación del sector directivo podría ser susceptible de una evaluación positiva. En efecto, en el marco de la auspicada flexibilización del "mercado de los directivos"16, la plena movilidad entre las distintas áreas de la Administración Pública podría favorecer una utilización simplificada y más economica de dicho colectivo. Además, este nuevo sistema, por un lado, consentiría a las Administraciones elegir los directivos en el ámbito de una platea de profesionalidades mucho más amplia y heterogénea $\mathrm{y}$, por otro lado, permitiría a los dirigentes crecer profesionalmente, más allá de la sola Administración de pertenencia.

De otra parte, no cabe discutir que la unificación del sector directivo puede dar lugar a varias perplejidades ${ }^{17}$. Antes de nada, cabe destacar que las profesionalidades que se encuentran en el ámbito de los distintos niveles de gobierno (estatal, regional, local) pueden referise unicamente a las caracteristicas peculiares de cada una de dichas administraciones, dada también la diversidad que existe entre las reglas que presidian el acceso a la función pública. Así pues, es difícil teorizar un sistema caracterizado por la plena movilidad entre directivos pertenecientes a sectores administrativos diferentes.

12 Vid. art. 2, Capo I, del citado esquema de decreto legislativo sobre el personal directivo público.

13 Vid., Ibidem, art. 28- quarter, Capo II.

14 VISCOMI, A. "La riforma dell'amministrazione del governo Renzi. Note brevi per una lettura del ddl 1577", en LPA, 3-4, 2014, p. 492.

15 La expresión es de D'ALESSIO, G., "La nuova disciplina della dirigenza nel disegno di legge sulla riorganizzazione delle amministrazioni pubbliche", en LPA, 2014; vid., en especial, el art. 2, Capo I, punto 7, del citado esquema de decreto legislativo sobre el personal directivo público.

16 Vid., por ejemplo, en general, sobre el concepto, de matriz europea, de "flexicurity" aplicado a las relaciones laborales, también en una perspectiva comparada", BALLESTRERO, M.V., "Declinazioni di flexicurity. La riforma italiana e la deriva spagnola, en DLRI, 2012, p. 441 e ss.

17 Vid., en este sentido, DEODATO, C., "Brevi Riflessioni sulla costituzionalità e sulla ragionevolezza di alcuni aspetti della riforma della dirigenza pubblica", en $L P A, 6,2014$. 
Bajo esta perspectiva, las previsiones en cuestión corren el riesgo de producir como único efecto inmediatamente tangible el de consentir al órgano político poder elegir libremente los directivos con una "discrecionalidad técnica" todavía mas amplia que en el pasado. Consecuentemente, es probable la violación de los principios establecidos por la jurisprudencia constitucional, ${ }^{18}$ la cual subraya como, al dia de hoy, dicha "discrecionalidad técnica" de los organos apicales continua siendo una fuente inagotable de problemas, tanto interpretativos como prácticos, porque tiende a construir una sistema de dirección publica muy atado a las veleidades del mundo de la politíca ${ }^{19}$.

Sin embargo, esta afirmación no debe impedir la búsqueda de un directivo profesional que sea capaz de mostrar sus resistencias a la implantación de un modelo precario de dirección (más bien "política" que "pública") basado en los cambios contundentes del partido que gobierna y en las afinidades ideológicas del personal directivo hacia el mismo.

La apuesta es desarrollar un enfoque empresarial del aparato administrativo, en el sentido de que el desafío para la politización de la clase directiva pasa por articular un personal de alta calidad que permitiría anticiparse a los cambios de Gobierno, desarrollar la capacidad de gestión y la integración ocupacional de los directivos públicos, e involucrarlos en la marcha de las empresas mediante un régimen jurídico inspirado al del derecho laboral vigente en el sector privado del trabajo.

De ahí, la importancia estratégica de la implicación de una Comisión independiente, instituida por la reforma Madia, encargada de verificar el cumplimiento exacto y puntual de las reglas que presidian la provisión de los cargos directivos, como se verá en adelante.

\section{NUEVAS REGLAS PARA LA ATRIBUCIÓN DEL CARGO DIRECTIVO Y EL PAPEL CRUCIAL DE LA COMISIÓN INDEPENDIENTE}

La reforma Madia establece nuevas reglas también en tema de atribución del cargo directivo ("incarico dirigenziale") 20 ; proponendo un modelo de dirección pública profesional en el cual el derecho de acceso en condiciones de igualdad a los puestos públicos juega un papel fundamental no solo en la fase del acceso, sino también en la fase sucesiva de la atribución de los cargos directivos. Además, el sistema de designación de los directivos ha de tener en cuenta una serie de "criterios generales

18 Vid., ex multis, Corte Cost. sentt. nn. 81/2013, 103/2007, 104/2007, 233/2006, 9/2006, 193/2002, 275/2001, 13/1996.

19 Vid. PATRONI GRIFFI, A., Dimensione costituzionale e modelli legislativi della dirigenza pubblica. Contributo ad uno studio del rapporto di "autonomia strumentale" tra politica e amministrazione, Napoli, Iovene, 2002.

20 Para un examen de estas previsiones, vid. BATTINI, S., "Una nuova stagione di riforme amministrative", en Giorn. dir. amm., 11/2014, p. 1018; VISCOMI, A., "Linee di riforma in materia di reclutamento e incarichi dirigenziali. Brevi note introduttive", en LPA, Vol. XVII, 1- 2014, p. 10. 
inspirados a los principios de publicidad, transparencia y merito", ${ }^{21}$ expresamente mencionados por las nuevas disposiciones legislativas.

Pues bien, a los efectos que aquí interesan, cabe hacer hincapié sobre dos preceptos de sumo interés: de una parte, la atribución a una Comisión independiente (Commissione per la dirigenza), compuesta por siete miembros y "operante en plena autonomía y con independencia de juicio y de evaluación"22, la tarea de verificar el respeto a los criterios de capacidad y mérito establecidos para la provisión del cargo directivo; $y$, de otra, la previsión según la cual el desempeño de los puestos directivos esté sujeto a plazo o a termino de una duración máxima de cuatro años con una sola posibilidad de renovación (por dos años más) subordinada a una especifica motivación y a la evaluación positiva de los resultados de su actividad ${ }^{23}$.

Con respecto al primer precepto, la Comisión, en especial, verifica la observancia de los requisitos y de los criterios de atribución de los cargos directivos de manera diferente según el tipo de cargo. Si se trata de un cargo apical, se requiere ex ante la evaluación de la Comisión, la cual procede pues con una verdadera "preselección" de los candidatos apropiados para los cargos directivos a atribuir. En cambio, en los demás casos, la Administración designa los candidatos y luego envia el listado de los designados a la Comisión que verifica, ex post, la conformidad de dicha elección con los requisitos y criterios previstos para la designación de los directivos ${ }^{24}$.

A la luz de lo dicho, el aporte de la Comisión al procedimiento de designación de los directivos se valora positivamente (en especial in apicibus), porque podría efectivamente contribuir a reforzar la distinción entre las funciones políticas y la gerencia administrativa que -como ya se ha apuntado- debe necesariamente caracterizar el colectivo en cuestión para asegurar una gestión efectiva y démocratica de la res publica. No obstante, debe reseñarse que la exigencia de fortalecer la distinción funcional entre tareas politícas y tareas administrativas del personal directivo público también a través del papel jugado por la Comisión independiente, podría peligrosamente prevalecer sobre la necesidad -igualmente importante- de asegurar el mantenimiento de cierta "fluidez" en el sistema de atribución de los cargos directivos, es decir que el mismo no sea afectado por una excesiva "burocratización"25.

Con referencia al segundo precepto, relativo a la duración de los cargos directivos, aun siendo cierto que la temporalidad constituye una garantía fundamental de autonomía del dirigente porque implica que él es el encargado de realizar sus funciones directivas por un período que no coincide plenamente con el mandato del

21 Vid. art. 4, Capo III, punto 2, lett.b) del esquema de decreto legislativo sobre el personal directivo público.

22 Vid. art 1, co. 1, lett.g), L. n. 124/2015 y art. 4, Capo III, punto 1 del esquema de decreto legislativo sobre el personal directivo público.

23 Ibidem, art. 19 quinquies, punto 2.

24 Ibidem, art. 19 ter, punto 5.

25 A. VISCOMI, "Linee di riforma in materia di reclutamento e incarichi dirigenziali"...cit., p. 9. 
gobierno, la previsión en virtud de la cual la finalización del plazo conlleva que el directivo pierde su puesto cualquiera que sean los resultados obtenidos (tanto positivos como negativos) de su actividad, podría acarrear una grave precarización del personal directivo público. No obstante, ello supone evitar la fácil tentación de llevar a cabo una "re-politicización" de los cargos directivos hacia donde transitaría la configuración de los puestos directivos como vitalicios según un sistema de designación basado en un "derecho débil" consistente en meras praxis administrativas, no orientadas hacia la armonización fuerte a través de instrumentos normativos que limiten la duración del cargo, con el rasgo significativo de que escaparía también al control judicial.

A este propósito, hay que añadir las previsiones contenidas en el citado esquema de decreto legislativo sobre el personal directivo público de 26 de agosto de 2016, las cuales -aunque (se repite) no hayan todavía entrado en vigor- si preveen la posibilidad de prorrogar el período del mandato. El nuevo art. 19-quinquies, en especial, tras confirmar la duración máxima de cuatro años del cargo directivo (salvo lo previsto por el art. 19-bis.4 sobre la atribución de cargos directivos a personal externo a la Administración Pública dentro del porcentaje establecido por la Ley) prevé una hipotésis de prorroga limitada a la circustancia de que ello sea necesario para completar el procedimiento de designación del nuevo cargo y, en todo caso, la prorroga no debe ser superior a noventa dias. Además, el mismo artículo prevé el cese de los cargos apicales ex art. 19 quarter. 1 tras noventa dias desde el inicio del nuevo gobierno. Se trata pues de un supuesto de spoil system. En base a ello, por lo que aquí interesa, conviene recordar que una de las cuestiones más controvertidas - posiblemente por el trasfondo político que subyace a la misma-, tanto en la doctrina como en la jurisprudencia constitucional italianas, tiene que ver con la posibilidad del órgano político que designa el directivo apical de exigir para el desempeño de funciones lato sensu políticas, ya sea como mérito o como requisito, un cierto nivel de confianza tanto en la fase del reclutamiento ("nomina a dedo"), como en el momento del cese ("spoil system" o "sistema de botín").

En especial, se ha profundizado en el tema concerniente al sistema de botín ${ }^{26}$, que nació con las experiencias de países anglosajones como los Estados Unidos ${ }^{27}$ y se

26 SETTIMI, R., "Dirigenza statale, incarichi ad esterni e spoils system (nota a Corte Costituzionale, sentenza 24 febbraio 2010, n. 81)",en LPA,2011, p. 128 e ss.; RUSCIANO, M., "Dirigenze pubbliche e spoils system", en www.astrid.online.it; DI NARDO, C., "Corte Costituzionale, sentenza 27 gennaio - 5 febbraio 2010, n. 34 (la Corte costituzionale torna (definitivamente?) sullo "spoils system" con una nuova pronuncia abrogativa", en LPA, 2010, p144 e ss.; SORDI, P., "La giurisprudenza costituzionale sullo spoils system e gli incarichi dirigenziali nelle pubbliche amministrazioni", en $L P A, 2009$, p.77 e ss.; D'ALESSIO, G., (a cura de), L'amministrazione come professione. I dirigenti pubblici tra spoils system e sevizio ai cittadini, Bologna, 2008; GRAGNOLI, E., "Lo spoils system e l'imparzialità del dipendente degli enti locali", en LPA, 2007, p. 25 e ss.; TALAMO, V., "Lo spoils system all' "italiana" fra legge Bassanini e legge Frattini", en LPA, 2003, p. 237 e ss.

27 Vid. KINGSLEY, D., Representative Burocracy, The Antioch Press, Ohio, 1944; GOODNOW, F. G., Politics and Administration, New York, 1900; WRIGHT MILLS, C., The power Elite, New York, 1956. 
tomó como paradigma en contextos comparados como el italiano o el español ${ }^{28}$, con perjuicio de que posteriormente se deteriorase el delicado equilibrio entre Política y Administración, haciendo los Ministros uso de espacios de discrecionalidad excesivamente amplios en la designación y en el cese de los directivos ${ }^{29}$.

De entre los innumerables pronunciamientos recaídos en relación a este tema se han identificado unas etapas que reflejan claramente la evolución seguida por el Tribunal Constitucional en la materia, a partir de los años $2006 / 2007^{30}$ hasta hoy en día ${ }^{31}$. Dichos pronunciamientos, como un "clímax descendente", parten de una primera etapa en la que se reconoce la legitimidad constitucional del sistema que consiente proveer y cesar empleos directivos en base a la confianza política, indicando la ventaja de la flexibilidad que -en ciertas circunstancias- presenta el reclutamiento y cese como consecuencia de la discrecionalidad política, hasta llegar a circunscribir el mecanismo del spoil system solo y exclusivamente a nivel apical, es decir en la Alta Administración, dada su ontológica continuidad con la cúspide política.

La doctrina y la jurisprudencia se han encargado de precisar cual es el alcance de esta interpretación.

Pues bien, la Corte Costituzionale, partiendo de una interpretación de los preceptos constitucionales habidos en materia, "desplazó" la presencia del spoil system principalmente hacia los niveles superiores de la Administración Pública (en los cuales es inevitable un minimum de confianza política), para evitar la paradoja de que mientras la función pública se profesionalizaba, se permitía que la dirección fuera servida por personas que no acreditaran esta profesionalización ${ }^{32}$.

28 Para ver como "vive" dicho fénomeno también en el ordenamiento jurídico español, vid. por todos, MARTÍN RETORTILLO, L., Méritos o botín y otros retos jurídicos de la democracia, Navarra, 2000.

29 HAM, C. -HILL, M., The policy process in the modern Capitalist State, tr. It. Introduzione all'analisi delle politiche pubbliche, Bologna, 1995.

30 Vid. Corte Cost. sentt. nn.. 233/2006; 103/2007; 104/2007.

31 Vid., por ejemplo, Corte Cost., sentt. nn. 390/2008; 168/2008; 81/2010; 224/2010; $124 / 2011 ; 246 / 2011 ; 152 / 2013 ; 27 / 2014 ; 24157 / 2015$.

32 Para un examen de la jurisprudencia constitucional dictada en materia, vid., MERLONI, F., "Lo spoils system è inapplicabile alla dirigenza professionale: dalla Corte nuovi passi nella giusta direzione (commento alle sentt. n. 103 e 104 del 2007)", en Le Regioni, 2007, 5, p.836 e ss.; DE GOTZEN, S., "Il bilanciamento tra spoils system e principio di distinzione tra politica e amministrazione nella dirigenza regionale", en Le Regioni, 2007, p. 848; SCOCA, F. G., "Politica e amministrazione nelle sentenze sullo spoils system", en Giur. Cost., 2007, 2, p.1615 e ss.; VIRGA, G., "Breve storia di un trapianto con crisi finale di rigetto", en www.lexitalia.it; JORIO, F., "Lo spoils system viene nuovamente ridisegnato dal Giudice delle Leggi con le sentenze nn. 103 e 104 del 2007: stabilizzazione della dirigenza e giusto procedimento", en www.federalismi.it; CASTRO, S., "Stop allo "Spoils system": problemi e prospettive. Le conseguenze dopo la Consulta, n. 103/2007", en Guida al Pubblico Impiego, 2007, 5, p.37 e ss.;VALENSISE, B., "La dirigenza amministrativa tra fiduciarietà della nomina ed il rispetto dei principi costituzionali del buon andamento e dell'imparzialità della pubblica amministrazione", en Giur. Cost., 2002, p.1193. 
Las previsiones contenidas en la reforma Madia reflejan dicha postura jurisprudencial y doctrinal, puesto que consienten el funcionamiento del mecanismo del spoil system -es decir la existencia de ceses discrecionales no basados en la responsabilidad por la gestión, sino en mera "perdida de confianza política"- solo con referencia a los cargos apicales.

\section{APUNTES SOBRE EL NUEVO SISTEMA DE ACCESO A LA FUNCIÓN DIRECTIVA}

Las modificaciones previstas en materia de acceso a la función directiva se caracterizan, principalmente, por dos novedades. La primera concierne a la adopción -en linea con la unificación del sector directivo- de un modelo unitario para todos los tipos de Administraciones Públicas, (nacionales, regionales y locales): el "doble canal" del "curso-concurso" y del "concurso" 33 , que ya existía en el ámbito de las Administraciones estatales y de los entes públicos nacionales.

La segunda consiste en la previsión según la cual el acceso al séctor directivo está subordinado no solo a un período de prueba, sino además a una verificación ulterior sobre la idoneidad del candidato en relación con la adquisición definitiva de la respectiva función directiva.

Sin embargo, hay que distinguir entre los candidatos que aprueban el "cursoconcurso" y los que aprueban el "concurso".

Más específícamente, los candidatos que aprueban el "curso-concurso" no se integran directamente en el ámbito del personal directivo, sino que empiezan a trabajar como funcionarios, durante un período de tres años. Una vez finalizado dicho período, si los candidatos no obtienen una "evaluación positiva", se quedan como funcionarios y no alcanzan el nivel directivo ${ }^{34}$.

En cambio, los candidatos que aprueban el "concurso" acceden a la función directiva a través de una relación de trabajo a tiempo definido, debiendo hacer, después de tres años, un "examen de confirmación" para pasar a ser directivos. Si suspenden dicho examen, se quedan como funcionarios ${ }^{35}$.

Dichas previsiones no nos permiten afirmar que la nueva regulación se sitúa en un entorno mucho más sensato que la situación previa, puesto que -aunque es cierto que la idoneidad en relación con la función directiva se presenta como criterio determinante para la configuración, en concreto, del singular puesto de trabajo- todos estos examenes y pruebas sucesivas a la primera selección podrían burocratizar excesivamente el procedimiento de acceso a la función directiva.

\footnotetext{
33 Vid. art. 3 lett. a), del esquema del decreto legislativo sobre el personal directivo público.

34 Ibidem, vid. art. 3 lett. b).

35 Ibidem, vid. art. 3 lett c)
} 
Además, esto chocaría con la "lucha contra el envejecimiento" del personal de las Administraciones Públicas formalmente perseguida por la reforma, puesto que el nuevo sistema consentiría el acceso a la función directiva solo a personal de edad avanzada. De hecho, al tiempo necesario para obtener la graduación y realizar los cursos post lauream se añaden también los años de prueba requiridos después de aprobar la oposición y los años necesarios para la preparación de la sucesiva verificación de idonead en relación con el respectivo puesto directivo.

Otro aspecto de criticidad es el hecho de que contratar los candidatos que aprueban el "curso-concurso" como "funcionarios" (y no directamente como directivos) y los que aprueban el "concurso" con un contrato a tiempo definido (y no a tiempo indefinido) puede dar lugar a una peligrosa precarización del personal directivo, destacando además cierta incertidumbre en relación con el régimen jurídico aplicable (tratamiento ecónomico y normativo, duración, etc.) en caso de suspensión del sucesivo "examen de confirmación".

Así pues -como ha sido observado por la doctrina- en lugar de subordinar el acceso a la función directiva a una especie de "doble escrutinio" del cual depende el propio "futuro laboral" del candidato, se podría enfatizar la importancia de la "formación continua" ("on the job") del personal directivo sobre todo durante los primeros años de actividad laboral, cuyos resultados podrían ser evaluados por algun organismo independiente ${ }^{36}$.

De esta manera, se evitaría la realización de los examenes sucesivos al primer escrutinio para no dar lugar a inútiles duplicidades y se valoraría no solo la preparación meramente teórica necesaria para aprobar el correspondiente examen, sino tambien el aspecto práctico del desarollo, en concreto, de la función directiva en su totalidad.

\section{ALGUNOS “RETOQUES” AL SISTEMA DE RESPONSABILIDAD GERENCIAL DE LOS DIRECTIVOS PÚBLICOS}

La reforma Madia vuelve a retocar el sistema de "responsabilidad gerencial o directiva", sin realmente introducir importantes novedades al respecto, excepto la previsión según la cual "habrá que limitar la responsabilidad disciplinar a los comportamientos efectivamente imputables a los directivos" ${ }^{37}$, lo cual podría determinar una todavía mayor "irresponsabilidad" de los órganos políticos.

De ahí la importancia estratégica no solo de la implicación de la dirigenza y su corresponsabilidad en la gestión administrativa, sino también de la definición de un marco de responsabilidades del directivo público frente a la organización, previsto por el art. 21 del D.lgs. 165/2001, en su nueva versión ofrecida por el esquema de decreto legislativo del 26 de agosto de 2016, que fundamenta su racionalidad en una "logica

36 D'ALESSIO, G., "La nuova disciplina della dirigenza nel disegno di legge”..cit., p. 6.

37 Vid. art.11.1,lett. m., L. 124/2015. 
de resultados", por lo que si un directivo no obtiene buenos rendimientos, performance útiles y resultados positivos en la organización que dirige, incurre en responsabilidad gerencial. Dicho marco de responsabilidades viene definido por un "contrato-programa", en donde se pactan una serie de metas y objetivos a alcanzar y se asignan los recursos (personales, materiales, financieros) necesarios para su cumplimiento.

Además, la última reforma somete las tareas del directivo público a un sistema de evaluación del desempeño y de los resultados de gestión que implica el aporte de la Comisión independiente en el caso de culpa in vigilando ${ }^{38}$.

No han sido "determinantes" pues los "retoques" aportados por la reforma Madia al sistema de responsabilidad gerencial, aunque constituye, sin duda, la pieza principal del modelo de directivo público profesional. En este sentido, la doctrina está todavía a la espera de un cambio estructural, que consienta el desarrollo de una combinación apropiada de flexibilidad (imbricada con la autonomía de la gestión derivada de las competencias propias o delegadas de que debe disponder todo directivo público) y estabilidad democrática (racionalizada por la definición precisa de los objetivos de la organización en un contrato de gestión) y subraya la necesitad de promover, a través del dialogo con los agentes sociales, una reforma del marco regulador de su relación de trabajo encentrada sobre el sistema de responsabilidad gerencial ${ }^{39}$.

A este respecto y a modo de reflexión general, cabe recordar que la exigencia prioritaria de asegurar un "itinerario" de autonomía al personal directivo respecto a la Política, necesita de medios determinados, entre los cuales destaca el que concierne a aquella responsabilidad "propia" y "pública" de la cual hablaba Pérsico hace ya un $\operatorname{siglo}^{40}$. Responsabilidad que tiene que ser, por un lado, "propia" porque el funcionario público "debe responder directamente" 41 de sus propias actuaciones y, por otro lado, "pública" porque debe resultar evaluable a través de procedimientos objetivamente medibles y transparentes y entonces controlables por el "público".

En definitiva, se trata de añadir parámetros fijos y claros en un contexto de inseguridad o indeterminación jurídica en el que sólo algunos de estos puntos se han establecido, pero todavía queda una labor ardua para alcanzar el pleno y deseable desarrollo de una más completa democracia, abundando en la objetividad de la actividad (y de la correlativa responsabilidad) de la administración pública, especialmente en sus puestos directivos ${ }^{42}$.

38 Vid. art 5.1, lett. b) del esquema de decreto legislativo sobre el personal directivo público.

39 BORGOGELLI, F., "Valutazione e responsabilità della dirigenza pubblica: ancora una riforma", en $L P A, 5,2014$, p. 689 e ss.

40 PERSICO, F., Principii di diritto amministrativo, Napoli, 1866.

41 BATTINI, S., "L'autonomia della dirigenza pubblica e la «riforma Brunetta»: verso un equilibrio tra distinzione e fiducia?", en Giorn. dir. amm., n.1/2010, p.39 e ss.

42 BORGOGELLI, F., "La fatica di sisifo: la ricerca delle regole sulla valutazione e sulla responsabilità della dirigenza pubblica", en Rev. Giur. Lav., n. 3/2015, p. 538, donde el A., en especial, observa como «la busqueda de una relación virtuosa entre politíca y administración -funcionalmente destinada a la realización del interés público- parece todavía configurarse como una "fatica de Sisifo"». 


\section{A MODO DE CONCLUSIÓN}

Como ya se ha apuntado, a partir de los años noventa, la situación de la Administración Pública italiana ha experimentado una gran trasformación, de manera que una Administración moderna se responsabiliza de la prestación de una pluralidad de servicios con eficiencia y eficacia, en una lógica empresarial parecida a la lógica de la empresa privada. Se hace necesario pues un rediseño organizativo bastante complejo, que introduzca un instrumento intermedio entre políticos y funcionarios que les permita trabajar en la misma dirección, aportando bienestar a la ciudadanía. Este instrumento lo representa precisamente el personal directivo público cuya profesionalidad ha de inspirarse también en la profesionalidad típica del directivo de una empresa privada.

No obstante, sin lugar a dudas, la característica más sobresaliente del personal directivo público italiano es que aparece revestido de múltiples especialidades que alcanzan todos los aspectos fundamentales de su relación de trabajo (inicio, desarrollo, extinción) confiriéndoles a tales directivos un status singular respecto al dirigente del sector privado.

El punctum dolens de la cuestión, reside pues en la exigencia, no de "copiar" la disciplina de derecho privado y "pegarla" en el régimen de derecho público (lo que -como se ha dicho- sería imposibile dadas las especialidades que caracterizan ontológicamente el empleo público), sino de tomar como paradigma algunos principios y requisitos sobre los que se rige habitualmente el derecho laboral y trasladarlos a los niveles superiores de la Administración Pública: principios de mérito, capacidad, publicidad, transparencia tanto en el proceso de selección como en el cese; funciones directivas que se vinculan habitualmente con titulaciones académicas y experiencias de trabajo; régimen de responsabilidad directiva correlato a los resultados de gestión; independencia de quien (o quienes) tiene que evaluar dicha gestión.

Todo ello fundamentaría la legitimación del poder de la propia alta burocracia que, según un modelo administrativo profesional, debe residir esencialmente en el bagaje de competencias y experiencias técnicas, organizativas y también stricto sensu jurídicas, acumuladas por el personal directivo durante su formación y su carrera profesional $^{43}$. Además, es evidente que sin la colaboración de un personal directivo que tenga adecuadas capacidad de liderazgo, formación y habilidades específicas, y conozca los "engranajes burocráticos" del aparato administrativo, el vértice político se encuentra en la imposibilidad práctica de conducir la compleja maquinaria jurídicoadministrativa en la dirección perseguida por el Gobierno ${ }^{44}$.

43 Es decir, en lo que Max Weber define como "saber de servicio", que - como es bien sabidoconsiste en las competencias adquiridas por la burocracia durante su servicio, lo cual debe llegar a ser su "caballo de batalla" en las relaciones (internas y externas) con el vértice político de la Administración Pública. Vid. WEBER, M., Wirtschaft und Gesellschaft, Tübingen, Mohr, 1922, trad. It. Economia e società, vol. I, Milano, 1968, p. 219.

44 De esta manera, la relación entre estas dos esfera jurídicas de acción (Política y Administración) dejaría de estar caracterizada únicamente por vínculos personales, y 
Desde esta perspectiva, el legislador de esta última reforma no se ha mostrado plenamente fiel a este propósito de inspirar el empleo público en la normativa específica del sector privado ${ }^{45}$, a pesar de que ello sea a menudo "declamado" como una operación clave para alcanzar la auspiciada profesionalización del personal directivo. En este sentido, la reforma Madia parece moverse más bien en una logíca parecida a aquella de la "re-publicización" del empleo publico que ha inspirado las reformas sucesivas a la privatización, reduciendo, cada vez mas, los espacios de maniobra de la negociación colectiva para volver a atribuir mayor poder regulador a la Ley ${ }^{46}$.

El riesgo que corre todo ello consiste en una posible disminución de tutelas efectivas y de autonomía de acción del dirigente ${ }^{47}$, que resulta "ahogado" por una híper regulación normativa que -paradojicamente y en el sentido opuesto al que (formalmente) inspira la reforma- podría reforzar la arbitrariedad de las decisiones políticas.

Sin embargo, hay que destacar que algunas previsiones, como por ejemplo las dedicadas a la configuración de una Comisión ad hoc que verifique el cumplimiento de los requisitos para la designación del cargo directivo, aunque -como se ha vistopueda dar lugar a excesos burocráticos o juegos de poder donde la burocracía abusa de su control para defender sus propias opciones de política, responde, sin lugar a dudas, a un modelo de directivo profesionalizado.

A la luz de lo expuesto hasta ahora, es evidente que la situación italiana actual se carácteriza por la presencia de un panorama de particular dispersión normativa (corroborado por las últimas reformas), que, obviamente, deriva también de la circustancia de que nos encontramos en una época de constantes y crecientes modificaciones jurídicas, debidas principalmente a la crisis económica, lo cual se refleja visiblemente en la Administración Pública italiana, sobre todo a la luz de los

consistiría en un "sistema equitativo de control de la gestión pública"; vid., en este sentido, FORTE, P., Il Principio di distinzione tra Politica e Amministrazione, Torino, 2005; D'AURIA, G., "I Controlli", en CASSESE, S., Trattato di Diritto Amministrativo - Parte generale, Milano, 2000, p. 1271.

45 En este sentido, vid. ZOPPOLI, L., "Alla ricerca di una nuova riforma della dirigenza pubblica: reclutamento e incarichi tra confusione e rilegificazione", en Riv. Giur. Lav., n. 3, 2015, p. 519.

46 A este proposito, cabe reseñar que la inseguridad jurídica que provocó este ulterior cambio de escenario -debido, en especial, al D.lgs. 150/2009 ("reforma Brunetta")- fue determinada sobre todo por la operación de "rilegificazione" de la materia, realizada básicamente a través, por un lado, de la previsión de unas normas inderogables sobre aspectos fundamentales de la relación de trabajo (sanciones disciplinares, movilidad, progresión de carrera, etc.) y, por otro lado, de la exclusión de la contratación colectiva de otros aspectos significativos, como los concernientes a la designación o al cese; vid., en este sentido, por todos, BELLAVISTA, A.- GARILLI, A., "Riregolazione legale e decontrattualizzazione: la neoibridazione normativa del lavoro nelle pubbliche amministrazioni", en $L P A, 1,2010$, p. 1 e ss.

47 Lo cual ha sido subrayado también por el Consiglio di Stato en los citados dictamenes del 14 de octubre de 2016 y del 9 de enero de 2017. 
recientes y repentinos cambios políticos que se han resumido anteriormente en este trabajo.

No obstante, mal que nos pese, siempre van a existir sectores de la Administración Pública que pasen por periodos difíciles y dificultades, o necesiten reestructurar su plantilla. Es cierto que resulta especialmente complicado "racionalizar" los procesos de reestructuración de plantillas procurando conciliar la "razón económica" con la "razón social", asegurando protección no solo a los trabajadores que forman parte de la organización productiva, sino además a los ciudadanos, cuyos intereses generales son constitucionalmente tutelados. Pero es cierto también que, hoy en día, los procesos de reestructuración se han convertido en una realidad permanente, no marginal ni coyuntural.

Dicha situación de confusión y ambigüedad no se limita a evidenciar la deficiente técnica jurídica del legislador (ya probablemente evidenciada por la citada sentencia n.251/2016 del Tribunal Constitucional italiano), sino que conlleva problemas prácticos relevantes de los cuales ya no se puede huir, siendo el ciudadano quien paga, en última instancia, los fallos de quienes dirigen las Administraciones Públicas.

\section{BIBLIOGRAFÍA}

BALLESTRERO, M.V., "Declinazioni di flexicurity. La riforma italiana e la deriva spagnola", in DLRI, 2012.

BATTINI, S., "L'autonomia della dirigenza pubblica e la "riforma Brunetta»: verso un equilibrio tra distinzione e fiducia?", en Giorn. dir. amm., n.1/2010.

BATTINI, S., “Una nuova stagione di riforme amministrative”, en Giorn. dir. amm., $11 / 2014$.

BATTINI, S., CASSESE, S. (a cura de), Dall'impiego pubblico al rapporto di lavoro con le pubbliche amministrazioni, Milano, 1997.

BELLAVISTA, A.- GARILLI, A., "Riregolazione legale e decontrattualizzazione: la neoibridazione normativa del lavoro nelle pubbliche amministrazioni", en LPA, 1 , 2010.

BORGOGELLI, F., “La fatica di sisifo: la ricerca delle regole sulla valutazione e sulla responsabilità della dirigenza pubblica", en Rev. Giur. Lav., n. 3/2015.

BORGOGELLI, F., "Valutazione e responsabilità della dirigenza pubblica: ancora una riforma", en $L P A, 5,2014$.

BOSCATI, A., "La politica del governo Renzi per il settore pubblico tra conservazione e innovazione: il cielo illuminato diverrà luce perpetua?", en LPA, 2014.

CARINCI, F., “Sisifo riformatore: la dirigenza”, en LPA, 2001.

CASSESE, S., “Il sofisma della privatizzazione del pubblico impiego”, en Riv. it. dir. lav., I, 1993.

CASTRO, S., "Stop allo "Spoils system": problemi e prospettive. Le conseguenze dopo la Consulta, n. 103/2007", en Guida al Pubblico Impiego, 5, 2007. 
CECORA, G., D'ORTA, C., La riforma del pubblico impiego, Bologna, 1993.

CLARICH, M., IARIA, D., La riforma del pubblico impiego, Rimini, 1994.

D’ALESSIO, G., “La nuova disciplina della dirigenza nel disegno di legge sulla riorganizzazione delle amministrazioni pubbliche", en LPA, 2014.

D'ALESSIO, G., (a cura de), L'amministrazione come professione. I dirigenti pubblici tra spoils system e sevizio ai cittadini, Bologna, 2008.

D'AURIA, G., “I Controlli”, en CASSESE, S. (dirigido por), Trattato di Diritto Amministrativo - Parte generale, Milano, 2000.

DE GOTZEN, S., "Il bilanciamento tra spoils system e principio di distinzione tra politica e amministrazione nella dirigenza regionale", en Le Regioni, 2007.

DEODATO, C., "Brevi riflessioni sulla costituzionalità e sulla ragionevolezza di alcuni aspetti della riforma della dirigenza pubblica", en LPA, n. 6, 2014.

DI CERBO, V., “La cosiddetta “privatizzazione del pubblico impiego"”, en Lav. prev. oggi, 1993.

GHEZZI, G., La legge delega per la riforma del pubblico impiego, en Riv. Giur. Lav., I, 1992.

DI NARDO, C., “Corte Costituzionale, sentenza 27 gennaio - 5 febbraio 2010, n. 34 (la Corte costituzionale torna (definitivamente?) sullo "spoils system" con una nuova pronuncia abrogativa", en LPA, 2010.

D'ORTA, C., MEOLI, C., La riforma della dirigenza pubblica, Padova, 1994.

FERRANTE, V., “Fare i conti senza l'oste: le Regioni, la riforma Madia e la Corte Costituzionale", en www.ipsoa.it, 3 de diciembre de 2016.

D'ORTA, C., “Gli incarichi dirigenziali nello Stato dopo la legge 145/2002", en LPA, 2002.

FORTE, P., Il Principio di distinzione tra Politica e Amministrazione, Torino, 2005.

GARILLI, A., "La privatizzazione del lavoro nelle pubbliche amministrazioni e l'art.97 Cost.: di alcuni problemi e dei possibili rimedi”, en Riv. Giur. Lav., I, 2007.

GARILLI, A.,"Profili dell'organizzazione e tutela della professionalità nelle pubbliche amministrazioni”, en Giorn. Dir. Lav. Rel. Ind., 2004.

GIANNINI, M. S., "Per la privatizzazione del rapporto di pubblico impiego", en Scritti in onore di Mario Nigro, II, Milán, 1991.

GOODNOW, F. G., Politics and Administration, New York, 1900.

GRAGNOLI, E., “Lo spoils system e l'imparzialità del dipendente degli enti locali”, en LPA, 2007.

HAM, C. - HILL, M., The policy process in the modern Capitalist State, tr. It. Introduzione all'analisi delle politiche pubbliche, Bologna, 1995.

JORIO, F., “Lo spoils system viene nuovamente ridisegnato dal Giudice delle Leggi con le sentenze nn. 103 e 104 del 2007: stabilizzazione della dirigenza e giusto procedimento", en www.federalismi.it 
KICKERT, W.M., (dirigido por), The Study of Public Management in Europeand the US, London, 2008.

KINGSLEY, D., Representative Burocracy, The Antioch Press, Ohio, 1944.

LISO, F., “La privatizzazione dei rapporti di lavoro", en CARINCI, F. (dirigido por), II lavoro alle dipendenze delle amministrazioni pubbliche (dal d.lgs. 29/1993 alla finanziaria del 1995). Commentario, Milano, 1995.

MARSHALL, J., "La Corte Costituzionale, senza accorgersene, modifica la forma di Stato?", en Giorn. dir. amm., 6/2016.

MARTÍN RETORTILLO, L., Méritos o botín y otros retos jurídicos de la democracia, Navarra, 2000.

MERLONI, F., "Lo spoils system è inapplicabile alla dirigenza professionale: dalla Corte nuovi passi nella giusta direzione (commento alle sentt. n. 103 e 104 del 2007)", en Le Regioni, 5, 2007.

NAPOLI, M. - GARILLI, A., La terza riforma del lavoro pubblico tra aziendalismo e autoritarismo, Padova, 2013.

PATRONI GRIFFI, A., Dimensione costituzionale e modelli legislativi della dirigenza pubblica. Contributo ad uno studio del rapporto di "autonomia strumentale" tra politica e amministrazione, Napoli, 2002.

PINELLI, C., “Commento agli artt. 97 e 98”, en BRANCA, G.- PIZZORUSSO, A. (dirigido por), Commentario alla Costituzione, Bologna-Roma, 1994.

PENSABENE LIONTI, G, "La riforma della dirigenza pubblica: prospettive ambiziose e risultati deludenti", en www.adapt.it, 9 de enero de 2017.

PERSICO, F., Principii di diritto amministrativo, Napoli, 1866.

POLLIT, C. - BOUCKAERT, G., Public Management Reform, Oxford, 2011.

RUSCIANO, M., “Dirigenze pubbliche e spoils system”, in www.astrid.online.it

SCOCA, F. G., "Politica e amministrazione nelle sentenze sullo spoils system”, en Giur. Cost., 2, 2007.

SETTIMI, R., "Dirigenza statale, incarichi ad esterni e spoils system (nota a Corte Costituzionale, sentenza 24 febbraio 2010, n. 81)", en LPA,2011.

SORDI, P., "La giurisprudenza costituzionale sullo spoils system e gli incarichi dirigenziali nelle pubbliche amministrazioni", en LPA, 2009.

TALAMO, V., “Lo spoils system all' “italiana” fra legge Bassanini e legge Frattini”, en LPA, 2003.

VALENSISE, B., La dirigenza statale alla luce delle disposizioni normative contenute nella legge 15 luglio 2002, n. 145, Torino, 2002.

VALENSISE, B., “La dirigenza amministrativa tra fiduciarietà della nomina ed il rispetto dei principi costituzionali del buon andamento e dell'imparzialità della pubblica amministrazione", en Giur. Cost., 2002.

VIRGA, G. "Breve storia di un trapianto con crisi finale di rigetto", en www.lexitalia.it

VIRGA, P., Il pubblico impiego dopo la privatizzazione, Milano, 1993. 
VISCOMI, A. “La riforma dell'amministrazione del governo Renzi. Note brevi per una lettura del ddl 1577", en LPA, 3-4, 2014.

VISCOMI, A., "Linee di riforma in materia di reclutamento e incarichi dirigenziali. Brevi note introduttive", en LPA, Vol. XVII, 1- 2014.

WEBER, M., Wirtschaft und Gesellschaft, Tübingen, Mohr, 1922, trad. It. Economia e società, vol. I, Milano, 1968.

WRIGHT MILLS, C., The power Elite, New York, 1956.

ZOPPOLI, L., “La dirigenza nel pubblico impiego 'privatizzato'”, en RUSCIANO, M. ZOPPOLI, L. (a cura de), L'impiego pubblico nel diritto del lavoro, Torino, 1993.

ZOPPOLI, L., “Alla ricerca di una nuova riforma della dirigenza pubblica: reclutamento e incarichi tra confusione e rilegificazione", en Riv. Giur. Lav., n.3, 2015. 